\title{
Catalytic RNA world relics in Dicer RNAs
}

\author{
Sayak Ganguli*, Dey S.K., Priyanka Dhar, Protip Basu, Paushali Roy and Abhijit Datta \\ DBT-Centre for Bioinformatics, Presidency College, Kolkata, sayakbif@yahoo.com
}

\begin{abstract}
RNA interference (RNAi) is a naturally occurring phenomenon of RNA-mediated gene silencing that is highly conserved among multicellular organisms. In the first step of the pathway, long doublestranded RNA molecules are chopped into shorter duplexes with 2 nucleotide overhangs at both 3 ' ends by an endonuclease dubbed Dicer, the structure of which has been solved only recently. This results in the formation of small 21 nucleotide long RNAs, aptly named small or short interfering RNAs (siRNAs), which are incorporated into a multimeric protein complex, the RNA-induced silencing complex (RISC). One of the two-siRNA strands guides RISC to a complementary RNA. After hybridization the endonucleolytic "slicer" activity of RISC cleaves the target RNA, thus preventing its translation. While long double-stranded RNA molecules can be employed to induce RNAi in lower eukaryotes, siRNAs being 21 nucleotides in length have to be used for gene silencing in mammalian cells in order to prevent the activation of an unspecific interferon response [1]. In contrast to siRNAs, however, miRNAs are capable of inhibiting translation of the targeted mRNA without degrading it (at least in mammalian cells)[2-4]. The need for in silico analysis of the components of the RNA interference pathway arises from the fact that very little is known about the structural and interacting properties of the components. With the above background the analysis was performed to identify putative catalytic motifs in the mRNA of the DICER enzyme.
\end{abstract}

Key Words: RNAi, Drosha, RISC, miRNA, ribozymes, motifs

\section{Introduction}

Over the past two decades it has become clear that a variety of the RNA molecules have important or essential biological functions in cells. RNA is proficient at forming complex and varied tertiary structures as revealed by high resolution structures of a handful of RNAs. The secondary and tertiary structures of RNA are key for understanding their biological activity. Motifs that stabilize RNA 3D folds are relatively small and often involve backbone functional groups, making them impossible to detect even when in large families of secondary structures. Tetraloops and their receptors, $U$ - turns, dinucleotide platforms, ribose zippers and $S-$ turns all consist of $4-11$ nucleotides and occur within a variety of sequence contexts. In addition, non - canonical base pairs often create context dependent helical geometries or surfaces used in RNA - RNA and RNA - Protein recognition. One of the most essential structures of RNA is the RNA hairpin. It can guide RNA folding, determine interactions in a ribozyme, protect messenger RNA from degradation, serve as recognition motif for RNA binding proteins and act as substrates for enzymatic reactions. Eukaryotic small RNAs of approximately 21-24 nucleotides function as guide molecules in a remarkably wide range of biological processes, including developmental timing and patterning, formation of heterochromatin, genome rearrangement, and antiviral defense [6-7]. They belong to at least two general classes, miRNA and siRNA. miRNAs (approximately 21-22 nucleotides) are found in plants and animals and are often phylogenically conserved within their respective kingdoms. These miRNAs are formed from a precursor which is transcribed from genes which are non protein coding. A part of this nascent precursor adopts a fold-back structure that interacts with a multidomain RNasellI-like enzyme termed DICER or DICER-LIKE (DCL1 in Arabidopsis), which catalyzes accurate excision of the mature miRNA[8]. The miRNAs then associate with ribonucleoprotein complexes that function to negatively regulate target genes controlling a range of developmental events, such as timing of cell fate decisions, stem cell maintenance, apoptosis, organ morphogenesis and identity, and polarity [6]. siRNAs are chemically similar to miRNAs, although in plants they typically range in size between 21 and 24 nucleotides [9-11]. They are associated with both post-transcriptional forms of RNA interference and transcriptional silencing involving chromatin modification [7]. siRNAs are processed from precursors containing extensive or exclusive doublestranded RNA (dsRNA) structure, such as transcripts containing inverted repeats or intermediates formed during RNA virus replication. siRNA precursors can also be formed by the activity of one or more cellular RNA-dependent RNA polymerases (RdRp), as was shown genetically in several screens for RNA silencing-defective mutants [13-16]. Arabidopsis plants contain at least three active RdRp genes, termed RDR1, RDR2, and RDR6 (also known as SDE1/SGS2) [14,15 and 19]. RDR6 is necessary for sense transgene mediated RNAi, but not for silencing of constructs that encode transcripts with hairpins containing extensive dsRNA structure [14,15 and 19]. In many animals, both miRNAs and siRNAs are formed by the activity of the same DICER enzyme [20-27], although in plants they are formed by distinct DCL activities [7]. Arabidopsis contains four DCL genes (DCL1 to DCL4), only one of which (DCL1) has been assigned a 
definitive function in small RNA biogenesis [2830]. Biochemical data indicate, however, that multiple DCL activities or pathways catalyze formation of siRNAs of small-sized (approximately 21 nucleotides) and large-sized (approximately 24 nucleotides) classes [11]. Endogenous siRNAs in plants arise from many types of retroelements and transposons, other highly repeated sequences, pseudogenes, intergenic regions (IGRs), and a few expressed genes [9,10 and 31]. Exogenous siRNAs can arise from both sense and hairpin transcriptforming transgenes and by viruses [32 and 33]. Both siRNAs and miRNAs function posttranscriptionally to suppress or inactivate target RNAs. siRNAs guide sequence-specific nucleolytic activity of the RNA-induced silencing complex to complementary target sequences. Among other proteins, RISCs contain ARGONAUTE (AGO) family members that likely bind siRNAs or target sequences [34]. In plants and insects, post-transcriptional RNAi serves as an adaptive antiviral defense response [35 and 36]. miRNAs are fully competent to guide nucleolytic function of RISC, provided that a target sequence with sufficient complementarity is available [37,38 and 11]. Many plant miRNAs function as negative regulators through this cleavage-type mechanism [10-11, 39-43]. In animals, the level of complementarity between target and miRNA sequences is generally low, which inhibits nucleolytic activity. Animal miRNAs suppress translation of target mRNAs [44 and 29]. Some plant miRNAs may also function as translational suppressors [45, 47]. siRNAs also guide chromatin-based events that result in transcriptional silencing. Two lines of evidence support this view. First, in Schizosaccharomyces pombe and Arabidopsis, endogenous siRNAs from repeated sequences corresponding to centromeres, transposons, and retroelements are relatively abundant $[10,31,46]$. RNAi-related factors (DICER, RdRp, and AGO proteins) are required to maintain $S$. pombe centromeric repeats and nearby sequences in a transcriptionally inactive, heterochromatic state. Mutants that lose RNAi component activities lose heterochromatic marks, such as histone $\mathrm{H} 3$ methylation at the $\mathrm{K} 9$ position (H3K9), as well as centromere function [16]. In plants, AGO4 is necessary to maintain transcriptionally silent epialleles of SUPERMAN. The ago4 mutants lose both cytosine methylation, particularly at nonCpG positions, and H3K9 methylation at SUPERMAN and other constitutive heterochromatic sites (the Arabidopsis thaliana short interspersed element 1 [AtSN1] locus) [48]. And, second, heterochromatin formation of nuclear DNA can be triggered, in a sequencespecific manner, by post-transcriptional silencing of cytoplasmic RNAs [49-51].

\section{Materials and Methods}

Since the work was performed absolutely in silico hence the materials are existing database sequences available at the public databases such as SWISS - PROT and GenBank of NCBI.

The Accession numbers of the sequences used are provided below:

- $\quad$ >gi|50897086|dbj|AB182481.1|

Tetrahymena thermophila DCL1 mRNA for Dicer-related RNase III protein Dcl1p.

- $\quad$ gi|86565498|ref|NM_068617.4| Caenorhabditis elegans Dicer Related Helicase family member (drh-1) (drh-1) mRNA, complete cds.

- $\quad$ >gi|89114031|gb|DQ398891.1| Drosophila melanogaster isolate Dicer-1 (Dcr1) gene.

- $\quad$ >gi|78099742|gb|DQ208406.1| Oryza sativa (indica cultivar-group) dicer-like protein mRNA.

- $\quad$ >gi|30677869|ref|NM_099986.2| Arabidopsis thaliana DCL1 (DICER-LIKE1); ATP-dependent helicase/ ribonuclease III (DCL1) mRNA, complete cds.

- >gi|125841883|ref|XM_678382.2| PREDICTED: Danio rerio Dicer1.

- $\quad>$ gi|117168270|ref|NM_148948.2| Mus musculus Dicer1.

- $\quad$ gi|29294650|ref|NM_177438.1| Homo sapiens Dicer1.

\section{Transcriptomic Analysis}

The mRNA sequences corresponding to the query sequences were derived using the BIOINFX server and were analyzed using the RNA analyzer tool at the Vienna RNA suite. The secondary structures of the snRNP motifs were modeled using the Quick Fold server.

\section{RESULTS AND DISCUSSION}

Secondary structures of the RNA sequences were obtained for all the sequences under study and the structures showed multiple bulges and stem loop structures. When these RNA secondary structures were analyzed using the Vienna RNA suite then multiple snRNP motifs were identified along with few regulatory protein binding motifs. The results of the analysis show that all dicer mRNAs form secondary structures resulting in higher stability and prevent themselves from being digested by cellular nucleases [53]. The presence of multiple sites for regulatory factor binding indicates that these areas may also serve as riboswitches or RNA aptamers which regulate when the mRNAs will be translated[54]. One of the most interesting observation is the fact that the mRNAs all posses multiple snRNP motifs which indicate that these might have catalytic properties. The Drosophila mRNA as indicated by the RNA analyzer tool does not possess a functional product, but posseses multiple stem loop structures in 
clusters - a property common to many catalytic RNA and group I introns. This is a breakthrough result as this proves beyond doubt that the RNAi machinery is nothing but RNA world relics. Probably in the ancient period they acted to regulate the production of proteins in a more active manner but with the gradual emergence of DNA as the genetic material their functions have become restricted towards the silencing of the expression of the unwanted mRNAs in the cell.

As evident from the secondary structure that there are multiple stem / hairpin loop structures in all the mRNAs. Most RNA secondary structures exhibit the presence of an RNA hairpin. This unique structure possesses the ability to guide RNA folding, determine interactions in a ribozyme, protect messenger RNA from degradation, serve as a recognition motif for RNA binding proteins or act as a substrate for enzymatic reactions [55]. Most of the regulatory snRNP motifs showed inclination towards purine - purine and pyrimidine - pyrimidine base pairing (FIG1) As no such regulatory mechanisms have been reported until now we can conclude that probably these stem loop structures play important roles in localization of the mRNAs and thus regulate translation [56].

\author{
List of abbreviations used \\ RNAi : RNA interference \\ siRNAs : small or short interfering RNAs \\ RISC : RNA-induced silencing complex \\ miRNAs : microRNAs \\ dsRNA : double-stranded RNA \\ RdRp : RNA-dependent RNA polymerases \\ IGRs : intergenic regions \\ AGO : ARGONAUTE \\ mRNA : messenger RNA
}

\section{Acknowledgement}

The authors are acknowledging the financial support from DBT, Govt. of India.

\section{References}

[1] Wallace M., Worrall E., Pettersson S., Hupp, T. R. \& Ball K. L. Molecular Cell 23, 251-263.

(2006)

[2] Wallace M. and Ball K.L. (2004) Mol Cell Biol 24, 5606-6619.

[3] Ball K.L., Lain S. Fahraeus R., Smythe C. and Lane D.P. (1997) Current Biology 7, 71-80.

[4] Behrens S. E., Tomei L. and De Francesco R. (1996) EMBO J. 15: 12-22

[5] Kato J. and Niitsu Y. (2002) Int. J. Hematol. 76: 208-212

[6] Carrington J.C. and Ambros V.(2003) Science 301 (5631): 336-338.

[7] Finnegan E. J. and Matzke M. A.(2003) Journal of Cell Science 116, 4689-4693.

[8] Denli A.M., Hannon G.J. (2003) Trends Biochem Sci 28(4):196-201.
[9] Hamilton A., Voinnet O., Chappell L. and Baulcombe D. (2002) EMBO J. 21: 4671-4679

[10] Llave C., Kasschau K.D., Rector M.A., and Carrington J.C. (2002a) Plant Cell 14: 1605-1619.

[11] Tang Guiliang Reinhrt, Brenda J., Bartel David P. and Zamore Phillip D. (2003) Genes and Development, vol. 17, no. 1, p. $49-63$

[12] Cogoni C., Macino G. (1999a) Current opinion in microbiology;2(6):657-62.

[13] Cogoni C., Macino G. (1999b) Nature;399(6732):166-9.

[14] Dalmay T., Hamilton A., Rudd S., Angell S. and Baulcombe D. (2000) Cell 101, 543553

[15] Mourrain P., Béclin C., Elmayan T., Feuerbach F., Godon C., Morel J.B., Jouette D., Lacombe A.M., Nikic S., Picault N., Rémoué K., Sanial M., Vo T.A., Vaucheret H. (2000) Cell 101:53342.

[16] Volpe T.A., Kidner C., Hall I.M., Teng G., Grewal S.I., Martienssen R.A. (2002) Science 297:1833-7.

[17] Smardon A.M., et al. (2000) Current Biology 10: R393-R394

[18] Yu D., Fan B., Macfarlane S. A. and Chen Z. (2003) Mol. Plant-Microbe Interact. 16: 206-216.

[19] Beclin C., Boutet S., Waterhouse P. and Vaucheret H. (2002) Curr. Biol. 12: 684688.

[20] Grishok A., Pasquinelli A.E., Conte D., Li N., Parrish S., Ha I., Baillie D.L., Fire A., Ruvkun G., Mello C.C. (2001) Cell.106:23-34.

[21] Ketting R.F., Fischer S.E., Bernstein E., Sijen T., Hannon G.J., Plasterk R.H. (2001) Genes \& Dev:15:2654-2659.

[22] Hutvagner G., McLachlan J., Pasquinelli A.E., Balint E., Tuschl T., Zamore P.D. (2001) Science. 293:834-838.

[23] Knight S.W., Bass B.L. (2001) Science:293:2269-2271.

[24] Provost P., Dishart D., Doucet J., Frendewey D., Samuelsson B. \& Radmark O. (2002a) EMBO Journal 21 5864-5874.

[25] Provost P., Silverstein R.A., Dishart D., Walfridsson J., Djupedal I., Kniola B., Wright A., Samuelsson B., Radmark O. \& Ekwall K. (2002b) PNAS :99 1664816653.

[26] Zhang H., Kolb F.A., Brondani V., Billy E. \& Filipowicz W. (2002) EMBO Journal 21 5875-5885.

[27] Myers J.W., Jones J.T., Meyer T. and Ferrell J.E. (2003) Nat. Biotechnol. 21: 324-328. 
[28] Park W., Li J., Song R., Messing J. and Chen X. (2002) Curr. Biol. 12: 14841495.

[29] Reinhart B.J., Weinstein E.G., Rhoades M.W., Bartel B. and Bartel D.P. (2002) Genes Dev. 16: 1616-1626.

[30] Schauer S.E., Jacobsen S.E., Meinke D.W. and Ray A. (2002) Trends Plant Sci. 7: 487-491.

[31] Mette M. F., Winden J. van der, Matzke M. and Matzke A. J.M.(2002) Plant Physiol, Vol. 130: 6-9.

[32] Hamilton A.J., Baulcombe D.C. (1999) Science: 29;286(5441):950-2.

[33] Mette M.F., Aufsatz W., van der Winden J., Matzke M., Matzke A.J.M. (2000) EMBO J 19: 5194-5201

[34] Carmell M.A., Xuan Z., Zhang M.Q., Hannon G.J. (2002) Genes Dev. 16(21): 2733--2742.

[35] Waterhouse P.M., Wang M.B. and Lough T. (2001) Nature 411, 834-842.

[36] Li X., Scuderi A., Letsou A., Virshup D.M. (2002) Mol. Cell. Biol. 22(11): 3674-3684.

[37] Hutvágner G. and Zamore P. D. (2002) Science 20: Vol. 297. no. 5589: $2056-$ 2060 ,DOI: $10.1126 /$ science. 1073827

[38] Doench J.G., Sharp P.A. (2004) Genes Dev.: 1. 18(5):504-11.

[39] Rhoades M.W., Reinhart B.J., Lim L.P., Burge C.B., Bartel B. and Bartel D.P. (2002) Cell 110: 513-520.

[40] Emery J.F., Floyd S.K., Alvarez J., Eshed Y., Hawker N.P., Izhaki A., Baum S.F., Bowman J.L. (2003) Curr Biol 13: 17681774

[41] Kasschau K.D., Xie Z., Allen E., Llave C., Chapman E.J., Krizan K.A., Carrington J.C. (2003) Dev Cell 4: 205-217

[42] Palatnik J.F., Allen E., Wu X., Schommer C., Schwab R., Carrington J.C., Weigel D. (2003) Nature 425: 257-263

[43] Xie Z., Johansen L.K., Gustafson A.M., Kasschau K.D., Lellis A.D., Zilberman D., Jacobsen S.E., Carrington J.C. (2004) PLoS Biol 2: 642-652

[44] Olsen P.H., Ambros V. (1999) Dev Biol. $15 ; 216(2): 671-80$.

[45] Aukerman M. J. and Sakai H.( 2003) The Plant Cell, Vol. 15:2730-2741.

[46] Reinhart B.J. and Bartel D. P.(2002) Science DOI: 10.1126/science.1077183.

[47] Chen X.(2003) Science DOI: 10.1126/science. 1088060.

[48] Zilberman D., Cao X. and Jacobsen S. E.(2003) Science : Vol. 299. no. 5607, pp. $\quad 716 \quad$ - 719.DOI: 10.1126/science.1079695
[49] Jones A.L., Hamilton A.J., Voinnet O., Thomas C.L., Maule A.J. (1999) Plant Cell. 11:2291-2230.

[50] Aufsatz W., Mette M.F., van der Winden J., Matzke A.J., Matzke M. (2002) Proc Natl Acad Sci USA; 99(Suppl 4):1649916506.

[51] Schramke V., Allshire R. (2003) Science.301:1069-1074.

[52] Ernst J., Ghanem L., Bar-Joseph Z., McNamara M., Brown J. (2009) PLoS ONE 4(10): e7469. doi:10.1371/journal.pone.0007469

[53] Mandal M. and Breaker R. R.(2004) Nature Reviews, Molecular Cell Biology, 5: 451463

[54] Svoboda P., Cara A. D.(2006) Cell Mol Life Sci, Vol. 63, No. 7-8: 901-908.

[55] Eeden F. J.M. van, Palacios I. M., Petronczki M., Weston M.J.D., and Johnston D.I St.(2001) The Journal of Cell Biology, 154 (3), 511-523.

\section{WEB REFERENCES}

[1] www.ncbi.nlm.nih.gov

[2] www.ebi.ac.uk.

[3] http://users.ugent.be/ mdgroeve/bioinfx/

[4] http://molbiol-tools.ca/RNA_analysis.htm

[5] http://wb2x01.biozentrum.uni-wuerzburg.de/ 
Table 1- DICER MOUSE (Length:9851, Origin: RNA, Promoter: none, Exon: 281- 6001, type-coding, PlyASignal:9832-9837)

\begin{tabular}{|c|c|c|c|}
\hline snRNP motifs & Position & Sequence & Quality \\
\hline snRNP motif: & 173 & gguucugg & + \\
\hline snRNP motif: & 766 & gguguuuga & + \\
\hline snRNP motif: & 790 & gauucuag & + \\
\hline snRNP motif: & 814 & aauuauga & + \\
\hline Put. sm site: & 853 & aauuuugg & ++ \\
\hline snRNP motif: & 1006 & gauugugg & + \\
\hline snRNP motif: & 1097 & gauuguaa & + \\
\hline snRNP motif: & 1174 & aguguugg & + \\
\hline snRNP motif: & 1439 & aguguuga & + \\
\hline snRNP motif: & 1933 & aauguuag & + \\
\hline snRNP motif: & 2002 & gaucuuaa & + \\
\hline snRNP motif: & 2575 & ggucuuaa & + \\
\hline snRNP motif: & 2945 & gauauuga & + \\
\hline Put. sm site: & 3062 & aauuuuga & ++ \\
\hline snRNP motif: & 3215 & gauguuga & + \\
\hline Put. sm site: & 3512 & gauuuuag & ++ \\
\hline snRNP motif: & 3812 & aguuauga & + \\
\hline snRNP motif: & 3920 & aauuauga & + \\
\hline snRNP motif: & 4590 & aguuuauag & + \\
\hline Put. sm site: & 4736 & gauuuuga & ++ \\
\hline snRNP motif: & 4814 & gguuucugg & + \\
\hline Put. sm site: & 5362 & gauuuugg & ++ \\
\hline snRNP motif: & 5422 & ggucuuga & + \\
\hline snRNP motif: & 5906 & gguguugg & + \\
\hline Put. sm site: & 6171 & aauuuugg & ++ \\
\hline Put. sm site: & 6270 & gguuuuaa & ++ \\
\hline Put. sm site: & 6291 & gguuuuugg & ++ \\
\hline snRNP motif: & 6340 & gguguuag & + \\
\hline Put. sm site: & 6548 & gauuuuga & ++ \\
\hline Put. sm site: & 6794 & gauuuuuaa & ++ \\
\hline snRNP motif: & 7648 & aguauuuaa & + \\
\hline snRNP motif: & 7668 & gauuguag & + \\
\hline snRNP motif: & 7771 & gaucuuuuga & + \\
\hline snRNP motif: & 7826 & gauugugg & + \\
\hline snRNP motif: & 7869 & gauuuauaa & + \\
\hline snRNP motif: & 8035 & aauauuga & + \\
\hline snRNP motif: & 8761 & gauuuguuga & + \\
\hline snRNP motif: & 8795 & aauguuuugg & + \\
\hline snRNP motif: & 9822 & aauuguag & + \\
\hline
\end{tabular}

Table 2- C elegans DICER (Length: 3281, Origin: RNA, Promoter: none, Exon: 7- 3066, type- coding, Poly A Signal: none)

\begin{tabular}{|l|l|l|l|}
\hline snRNP motifs & position & sequence & quality \\
\hline snRNP motif: & 390 & gguucugg & + \\
\hline snRNP motif: & 438 & aguucuga & + \\
\hline snRNP motif: & 1062 & gauucuga & + \\
\hline snRNP motif: & 1200 & gauuguaa & + \\
\hline snRNP motif: & 1791 & gguuuguaa & + \\
\hline snRNP motif: & 2052 & gauuucuaa & + \\
\hline Put. sm site: & 2157 & aguuuuga & ++ \\
\hline snRNP motif: & 2756 & gauucugg & + \\
\hline snRNP motif: & 2851 & aauugugg & + \\
\hline snRNP motif: & 3066 & gguauuuga & + \\
\hline snRNP motif: & 3142 & aguauuuga & + \\
\hline
\end{tabular}


Table 3- DICER HUMAN(Length: 10276, Origin: RNA, Promoter: none, Exon: 270- 6008, type-coding, Exon: 6360- 6565, type- coding, Poly A Signal 1: 6159- 6164, Poly A Signal 2: 6334- 6339)

\begin{tabular}{|c|c|c|c|}
\hline snRNP motif & position & sequence & quality \\
\hline snRNP & 708 & aauguuuuga & + \\
\hline snRNP & 755 & gguguuuga & + \\
\hline snRNP & 803 & aauuauga & + \\
\hline snRNP & 923 & aauucuuaa & + \\
\hline snRNP & 962 & ggucuuag & + \\
\hline snRNP & 995 & gauugugg & + \\
\hline snRNP & 1005 & gauugugg & + \\
\hline snRNP & 1086 & gauuguaa & + \\
\hline snRNP & 1172 & aguucugg & + \\
\hline snRNP & 1351 & aauuuguaa & + \\
\hline snRNP & 1479 & gauucuga & + \\
\hline snRNP & 1812 & gguguuga & + \\
\hline snRNP & 1922 & aauguuag & + \\
\hline Put. Sm site & 1950 & aguuuuga & ++ \\
\hline snRNP & 1991 & gaucuuga & + \\
\hline snRNP & 2315 & aguuguag & + \\
\hline snRNP & 2503 & aguguuuga & + \\
\hline Put. Sm site & 2564 & gguuuuaa & ++ \\
\hline snRNP & 2910 & gauauuga & 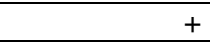 \\
\hline snRNP & 2934 & gauauuga & + \\
\hline Put. Sm site & 3051 & aauuuuga & ++ \\
\hline snRNP & 3231 & aaucuuuuga & 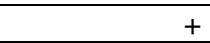 \\
\hline Put. sm site: & 3501 & gauuuuag & ++ \\
\hline snRNP & 3566 & aauuucuaa & + \\
\hline snRNP & 3801 & aguuauga & + \\
\hline snRNP & 4065 & gauucuga & + \\
\hline snRNP & 4355 & aauauuuga & + \\
\hline snRNP & 4591 & gauuuauag & + \\
\hline Put. sm site: & 4728 & gauuuuga & ++ \\
\hline Put. sm site: & 4737 & gauuuuga & ++ \\
\hline snRNP & 4816 & gguucugg & 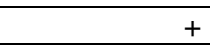 \\
\hline snRNP & 4845 & gguguuga & + \\
\hline Put. sm site: & 5241 & aauuuuga & ++ \\
\hline Put. sm site: & 5369 & gauuuugg & ++ \\
\hline snRNP & 5595 & gauucuga & + \\
\hline snRNP & 5640 & gauauuga & + \\
\hline snRNP & 5667 & gauauuuuuga & + \\
\hline snRNP & 5913 & gguguugg & + \\
\hline snRNP & 6062 & aauuauuuaa & + \\
\hline snRNP & 6091 & aguuguuag & + \\
\hline Put. sm site: & 6184 & aauuuugg & ++ \\
\hline snRNP & 6196 & aauuguag & 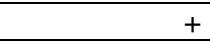 \\
\hline snRNP & 6315 & gguuuuguuuuuuuuuuuuuga & + \\
\hline snRNP & 6356 & aguguuag & + \\
\hline Put. sm site: & 6582 & gauuuuuaa & ++ \\
\hline snRNP & 6626 & aguuuucuga & + \\
\hline snRNP & 6694 & agucuuuaa & + \\
\hline snRNP & 6708 & gaucuuuuugg & + \\
\hline snRNP & 6903 & gaucuuugg & + \\
\hline snRNP & 7050 & gauuuuauaa & + \\
\hline Put. sm site: & 7221 & aauuuuga & ++ \\
\hline snRNP & 7326 & aguuucuuuaa & + \\
\hline Put. sm site: & 7436 & aauuuuaa & ++ \\
\hline snRNP & 7618 & gauucuga & + \\
\hline snRNP & 7785 & gguauuaa & + \\
\hline
\end{tabular}




\begin{tabular}{|l|c|c|c|}
\hline snRNP & 7820 & aaucuuaa & + \\
\hline Put. sm site: & 7857 & aguuuuuga & + \\
\hline snRNP & 7890 & aauauuuaa & ++ \\
\hline Put. sm site: & 7928 & gauuuuuuaa & + \\
\hline snRNP & 7976 & aauucuuga & + \\
\hline snRNP & 8045 & gauuuguga & ++ \\
\hline snRNP & 8193 & aguuauga & + \\
\hline Put. sm site: & 8393 & gauuuuaa & ++ \\
\hline snRNP & 8483 & gguuauga & ++ \\
\hline Put. sm site: & 8507 & gauuuuuaa & + \\
\hline Put. sm site: & 8801 & aauuuuuuaa & + \\
\hline snRNP & 8993 & gauuuaugg & + \\
\hline snRNP & 9016 & aauuauaa & + \\
\hline snRNP & 9109 & gauuuguuaa & + \\
\hline snRNP & 9280 & gauucuga & + \\
\hline snRNP & 9454 & aauauugg & ++ \\
\hline snRNP & 9562 & agucuuuaa & ++ \\
\hline snRNP & 9624 & gguuguuuuaa & + \\
\hline Put. sm site: & 9731 & aguuuuaa & + \\
\hline Put. sm site: & 9769 & gauuuuuaa & + \\
\hline snRNP & 9778 & aguuguugg & + \\
\hline snRNP & 10019 & aauuucugg & + \\
\hline snRNP & 10236 & aauuguag & + \\
\hline
\end{tabular}

Table 4- Danio rerio DICER (Length: 8708, Origin: RNA, Promoter: none, Exon: 158 - 4440, type- coding, Exon: 4495 - 5914, type- coding, Poly A-Signal: 7585 -7590)

\begin{tabular}{|l|l|l|l|}
\hline snRNP motif & position & sequence & quality \\
\hline Put. sm site: & 238 & gauuuuaa & ++ \\
\hline Put. sm site: & 781 & gguuuuuga & ++ \\
\hline snRNP & 838 & gauuuguga & + \\
\hline snRNP & 1031 & gauugugg & + \\
\hline snRNP & 1100 & aauuuucuaa & + \\
\hline snRNP & 1505 & gauucuga & + \\
\hline snRNP & 1627 & gguuguuuuaa & + \\
\hline snRNP & 1774 & agucuuga & + \\
\hline Put. Sm site: & 2057 & gauuuuga & ++ \\
\hline snRNP & 2664 & gauguuuugg & + \\
\hline snRNP & 2960 & gauauuga & + \\
\hline Put. sm site: & 3077 & aauuuuga & ++ \\
\hline snRNP & 3209 & aauguuaa & + \\
\hline Put. sm site: & 3358 & gauuuuag & ++ \\
\hline snRNP & 5259 & aguuucuugg & + \\
\hline snRNP & 5275 & aauucuag & + \\
\hline snRNP & 6072 & aguguuaa & + \\
\hline snRNP & 6320 & gguguugg & + \\
\hline snRNP & 6529 & aguuguga & + \\
\hline snRNP & 6696 & gauauuga & + \\
\hline snRNP & 6705 & gauauuuag & + \\
\hline Put. sm site: & 6757 & aguuuuga & ++ \\
\hline snRNP & 6962 & gauauuga & + \\
\hline Put. sm site: & 7579 & aauuuuaa & ++ \\
\hline snRNP & 8184 & aguuauugg & + \\
\hline Put. sm site: & 8438 & gguuuuga & \\
\hline
\end{tabular}


Table 5- Arabidopsis DICER (Length: 6188, Origin: RNA, Promoter: none, Exon: 381- 6104, type- coding,

\begin{tabular}{|l|l|l|c|}
\hline snRNP motif & Position Poly A Signal: none) & Quality \\
\hline SnRNP motif: & 371 & Sequence & + \\
\hline snRNP motif: & 758 & aauuaugg & + \\
\hline snRNP motif: & 813 & gguuuguag & + \\
\hline snRNP motif: & 832 & aauucuag & + \\
\hline snRNP motif: & 939 & gguuuauga & + \\
\hline snRNP motif: & 1017 & gguuauugg & + \\
\hline snRNP motif: & 1103 & gauguuaa & + \\
\hline snRNP motif: & 1421 & gguugugg & + \\
\hline snRNP motif: & 1870 & gguucuag & + \\
\hline snRNP motif: & 1916 & aauuuaugg & + \\
\hline snRNP motif: & 2586 & gguuuaugg & + \\
\hline snRNP motif: & 2787 & gauauuag & + \\
\hline snRNP motif: & 2846 & gaucuuag & ++ \\
\hline snRNP motif: & 3299 & aguuuauaa & + \\
\hline Put. sm site: & 3354 & aguuuguga & + \\
\hline snRNP motif: & 3404 & gauuuugg & + \\
\hline snRNP motif: & 3579 & gauucuuuuugg & + \\
\hline snRNP motif: & 3861 & gauguuga & + \\
\hline snRNP motif: & 4448 & gguauuag & + \\
\hline snRNP motif: & 4535 & gauucuuga & + \\
\hline snRNP motif: & 5010 & gguuguuag & + \\
\hline snRNP motif: & 5055 & aauguuaa & + \\
\hline snRNP motif: & 5164 & ggucuuga & + \\
\hline snRNP motif: & 5180 & aauuuguugg & + \\
\hline snRNP motif: & 5362 & ggucuugg & + \\
\hline snRNP motif: & 5441 & aauuuguga & + \\
\hline snRNP motif: & 6036 & aguucuugg & aguguuaa \\
\hline snRNP motif: & 6163 & gguuguag & + \\
\hline
\end{tabular}

Table 6- Drosophila DICER (Length: 4136, Origin: RNA, Promoter: none, Exons: none, Poly A Signal: none)

\begin{tabular}{|c|c|c|c|}
\hline snRNP & position & sequence & quality \\
\hline snRNP motif: & 23 & aauauuga & + \\
\hline Put. sm site: & 89 & aauuuuuuuuuuag & ++ \\
\hline snRNP motif: & 107 & aauuauuag & + \\
\hline snRNP motif: & 273 & gguuauga & + \\
\hline snRNP motif: & 488 & aauuuauga & + \\
\hline snRNP motif: & 606 & gaucuuga & + \\
\hline snRNP motif: & 615 & gauuauaa & + \\
\hline snRNP motif: & 698 & aauuuauaa & + \\
\hline Put. sm site: & 718 & gauuuuuuaa & ++ \\
\hline Put. sm site: & 729 & gauuuuuag & ++ \\
\hline snRNP motif: & 802 & aauuucuuuaa & 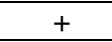 \\
\hline Put. sm site: & 879 & aauuuuga & ++ \\
\hline Put. sm site: & 942 & gauuuuga & ++ \\
\hline snRNP motif: & 966 & aaucuuuag & + \\
\hline Put. sm site: & 1029 & aauuuuag & ++ \\
\hline snRNP motif: & 1171 & aauuauaa & + \\
\hline snRNP motif: & 1521 & gauuuauuuuaa & 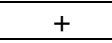 \\
\hline Put. sm site: & 1652 & gauuuuuga & ++ \\
\hline snRNP motif: & 1867 & aauuuguaa & + \\
\hline snRNP motif: & 1911 & gauuauuag & + \\
\hline snRNP motif: & 1941 & aauauuuaa & + \\
\hline snRNP motif: & 2264 & gauuguuag & + \\
\hline snRNP motif: & 2494 & aauauuuag & + \\
\hline snRNP motif: & 2626 & aaucuuuuuag & + \\
\hline
\end{tabular}




\begin{tabular}{|l|c|c|c|}
\hline snRNP motif: & 2672 & aguuguugg & + \\
\hline Put. sm site: & 2773 & aguuuuag & ++ \\
\hline snRNP motif: & 2866 & aauuauaa & + \\
\hline snRNP motif: & 2881 & aauuuuuauaa & ++ \\
\hline Put. sm site: & 3052 & aauuuuaa & + \\
\hline snRNP motif: & 3252 & gauuauuag & ++ \\
\hline Put. sm site: & 3268 & gauuuuugg & + \\
\hline Put. sm site: & 3293 & aauauuag & + \\
\hline snRNP motif: & 3457 & aauuuauag & + \\
\hline snRNP motif: & 3481 & aguucuag & + \\
\hline snRNP motif: & 3551 & gauuuaugg & + \\
\hline snRNP motif: & 3561 & gauucuga & + \\
\hline snRNP motif: & 3597 & aauuuaugg & + \\
\hline snRNP motif: & 3623 & aauuauuuuuaa & + \\
\hline snRNP motif: & 3680 & gauucuaa & + \\
\hline snRNP motif: & 3797 & aguauuuag & + \\
\hline snRNP motif: & 4076 & & \\
\hline
\end{tabular}

Table 7- Oryza sativa DICER (Length: 5193, Origin: RNA, Promoter: none, Exon 1: 287 - 1359, type-

\begin{tabular}{|c|c|c|c|}
\hline snRNP motif & Position & Sequence & Quality \\
\hline snRNP & 147 & gauguugg & + \\
\hline snRNP & 574 & aauauuuga & + \\
\hline snRNP & 633 & aauuuuauuuugg & + \\
\hline snRNP & 719 & gauuguga & + \\
\hline snRNP & 820 & aauuguga & + \\
\hline snRNP & 1025 & gaucuugg & + \\
\hline snRNP & 1091 & gauucuga & + \\
\hline snRNP & 1333 & aauuauga & + \\
\hline snRNP & 1359 & gguuuauga & + \\
\hline snRNP & 1372 & aauuguuaa & + \\
\hline snRNP & 2445 & aaucuuugg & + \\
\hline snRNP & 2639 & aguugugg & + \\
\hline Put. sm & 3219 & gauuuugg & ++ \\
\hline snRNP & 3693 & gguuauga & + \\
\hline snRNP & 3914 & aauuucuugg & + \\
\hline snRNP & 3976 & gauguuga & + \\
\hline snRNP & 4044 & aguuguaa & + \\
\hline snRNP & 4188 & gguucuugg & + \\
\hline snRNP & 4224 & aguauuuuuag & + \\
\hline Put. sm & 4240 & gauuuuaa & ++ \\
\hline snRNP & 4398 & aauuauag & + \\
\hline snRNP & 4504 & gaucuuaa & + \\
\hline snRNP & 4766 & aauuugugg & + \\
\hline
\end{tabular}




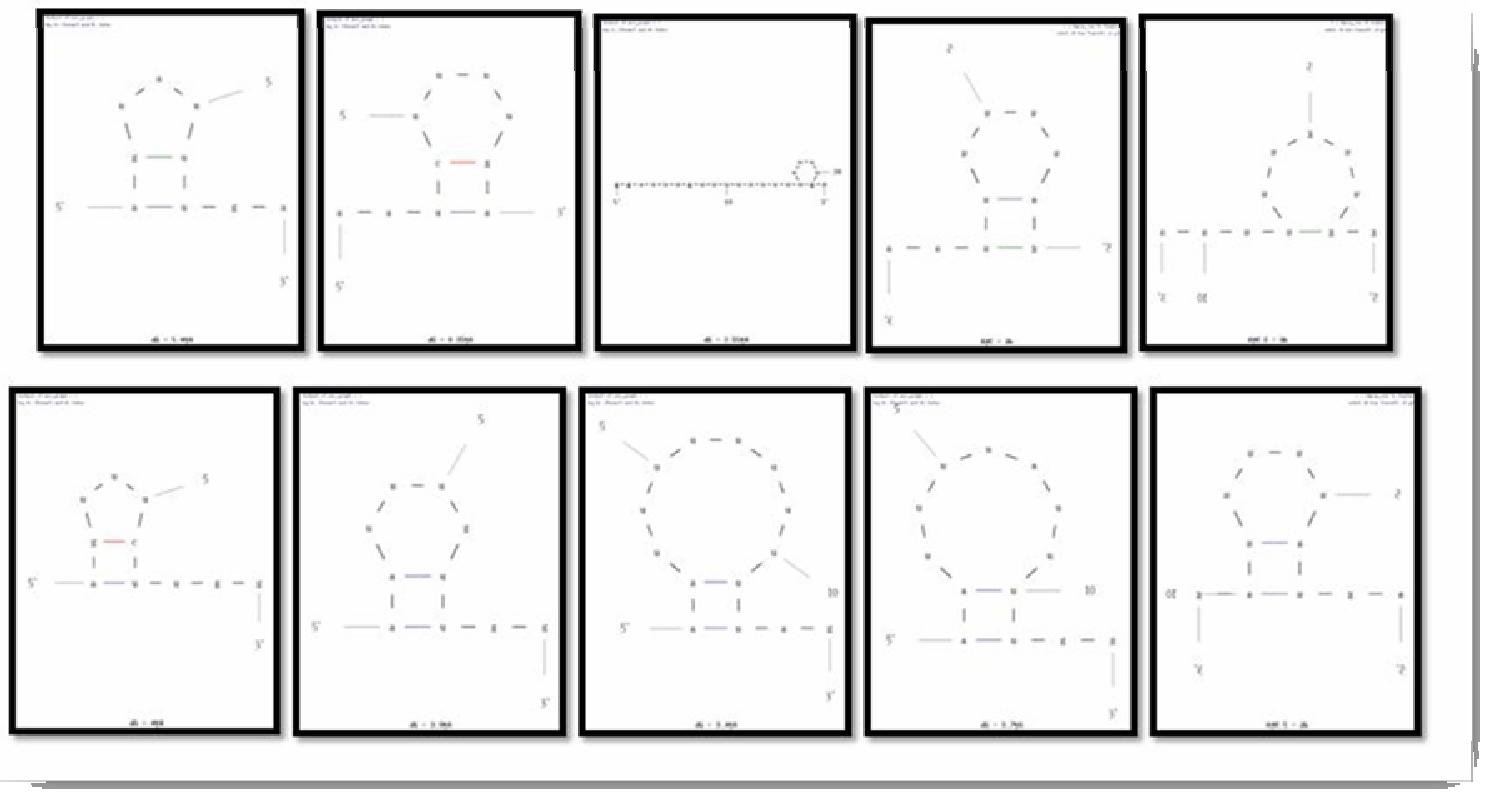

Fig. 1-Secondary Structure of important snRNP motifs in the DICER mRNAs 